\title{
Gastrin-Releasing Peptide Promotes Suprachiasmatic Nuclei Cellular Rhythmicity in the Absence of Vasoactive Intestinal Polypeptide- $\mathrm{VPAC}_{2}$ Receptor Signaling
}

\author{
Timothy M. Brown, Alun T. Hughes, and Hugh D. Piggins \\ Faculty of Life Sciences, University of Manchester, Manchester M13 9PT, United Kingdom
}

\begin{abstract}
Vasoactive intestinal polypeptide (VIP) and gastrin-releasing peptide (GRP) acting via the $\mathrm{VPAC}_{2}$ receptor and $\mathrm{BB}_{2}$ receptors, respectively, are key signaling pathways in the suprachiasmatic nuclei (SCN) circadian clock. Transgenic mice lacking the $\mathrm{VPAC}_{2}$ receptor $\left(\right.$ Vipr $2^{-/-}$) display a continuum of disrupted behavioral rhythms with only a minority capable of sustaining predictable cycles of rest and activity. However, electrical or molecular oscillations have not yet been detected in SCN cells from adult Vipr $2^{-1-}$ mice. Using a novel electrophysiological recording technique, we found that in brain slices from wild-type and behaviorally rhythmic Vipr $2^{-1-}$ mice, the majority of SCN neurons we detected displayed circadian firing patterns with estimated periods similar to the animals' behavior. In contrast, in slices from behaviorally arrhythmic Vipr $2^{-/-}$mice, only a small minority of the observed SCN cells oscillated. Remarkably, exogenous GRP promoted SCN cellular rhythms in Vipr $2^{-/-}$mouse slices, whereas blockade of $\mathrm{BB}_{2}$ receptors suppressed neuronal oscillations. In wild-type mice, perturbation of $\mathrm{GRP}-\mathrm{BB}_{2}$ signaling had few effects on $\mathrm{SCN}$ cellular rhythms, except when $\mathrm{VPAC}_{2}$ receptors were blocked pharmacologically. These findings establish that residual electrical oscillations persist in the SCN of Vipr2 ${ }^{-/-}$mice and reveal a potential new role for GRP- $\mathrm{BB}_{2}$ signaling within the circadian clock.
\end{abstract}

Key words: VIP; electrophysiology; hypothalamus; circadian; $\mathrm{BB}_{2}$ receptor; entrainment

\section{Introduction}

Individual neurons within the circadian clock of the suprachiasmatic nuclei (SCN) can act as autonomous oscillators, sustaining rhythmic clock gene expression (Quintero et al., 2003) and electrical activity (Welsh et al., 1995; Herzog et al., 1998) in culture. However, intercellular communication within the SCN is necessary to synchronize these cellular oscillators and produce a coherent circadian output (Yamaguchi et al., 2003; Herzog et al., 2004).

Vasoactive intestinal polypeptide (VIP) and its $\mathrm{VPAC}_{2}$ receptor are expressed by SCN neurons, and VIP-VPAC 2 signaling is one of the pathways via which SCN cellular activity is coordinated to ensure appropriate synchronization of the SCN pacemaker to cycles of light and dark (for review, see Hannibal and Fahrenkrug, 2003; Piggins and Cutler, 2003). Transgenic mice lacking $\mathrm{VPAC}_{2}$ receptor expression (Vipr $2^{-/}$) display a continuum of disrupted behavioral rhythms, ranging from complete arrhythmia to rhythmic individuals with altered periods (Hughes et al., 2004). Remarkably, this range of circadian phenotypes has not been detected in expression of core clock genes, immediate early gene, or

Received April 20, 2005; revised 0ct. 20, 2005; accepted 0ct. 21, 2005.

This work was supported by the Biotechnology and Biological Sciences Research Council (UK). We thank Rayna Samuels for technical assistance; Erik Herzog, Rob Lucas, Maria Canal, and Andrew Loudon for discussions and comments; Tony Harmar for supplying the initial breeding stock of Vipr2 ${ }^{-1-}$ mice; Jon Banks for assistance constructing equipment; and Patrick Robberecht for supplying the $\mathrm{VPAC}_{2}$ receptor antagonist. We also thank the anonymous reviewers for their helpful suggestions.

Correspondence should be addressed to Hugh D. Piggins, Faculty of Life Sciences, University of Manchester, Stopford Building, 0xford Road, Manchester M13 9PT, UK. E-mail: hugh.piggins@manchester.ac.uk.

D0I:10.1523/JNEUROSCI.3821-05.2005

Copyright $\odot 2005$ Society for Neuroscience 0270-6474/05/2511155-10\$15.00/0 spontaneous electrical activity of adult Vipr $2^{-1-}$ SCN (Harmar et al., 2002; Cutler et al., 2003; Hughes et al., 2004). In contrast, in culture, a small proportion of neonatal Vipr2 ${ }^{-1-}$ SCN neurons express circadian rhythms (Aton et al., 2005). It is unclear, therefore, whether previous studies used to assess circadian competence are sensitive enough to detect cellular rhythmicity in the adult Vipr2 ${ }^{-/-}$SCN. In addition, the factors that enable the expression of behavioral rhythms in some Vipr $2^{-1-}$ mice remain to be determined.

Many neurochemicals are present in the rodent SCN, and some SCN neurons containing VIP also coexpress peptide histidine isoleucine, GABA, and gastrin-releasing peptide (GRP) (Albers et al., 1991; Zoeller et al., 1992; Romijn et al., 1996, 1998; Aioun et al., 1998; Kawamoto et al., 2003). GRP, acting via its $\mathrm{BB}_{2}$ receptor, shares some of the resetting actions of VIP on the rodent SCN (Piggins et al., 1995; McArthur et al., 2000; Reed et al., 2001; Aida et al., 2002) and may potentiate the actions of VIP within the circadian pacemaker (Albers et al., 1991). However, unlike VIP, it is unclear what role, if any, GRP plays in sustaining cellular rhythms in the rodent SCN.

Here, we develop a novel and sensitive electrophysiological recording technique to reliably discriminate the electrical activity of single and multiple cells in brain slices prepared from adult mice. We show that in behaviorally rhythmic adult Vipr $2^{-/-}$and WT mice, the majority of SCN neurons detected display overt circadian oscillations. However, unlike WT mice, GRP-BB ${ }_{2}$ signaling was critical for the expression of SCN cellular oscillations in behaviorally rhythmic Vipr $2^{-/-}$mice. Very few rhythmic SCN 
neurons were detected in recordings from brain slices of behaviorally arrhythmic Vipr $2^{-/-}$animals, but stimulation of $\mathrm{BB}_{2}$ receptors with GRP promoted detectable cellular oscillations in SCN slices from these animals. These investigations show that cellular oscillations persist in the SCN of adult Vipr $2^{-/-}$mice and reveal a new, important role for $\mathrm{GRP}_{-} \mathrm{BB}_{2}$ signaling in promoting neuronal circadian rhythms in the absence of VIP-VPAC 2 signaling.

\section{Materials and Methods}

Animals. Adult male and female Vipr $2^{-1-}$ (University of Manchester stock derived from breeding pairs supplied by Prof. T. Harmar, University of Edinburgh, Edinburgh, UK) and WT (C57BL/6J) mice (Harlan, Bicester, UK) were group housed under a $12 \mathrm{~h}$ light/dark cycle (LD) at an ambient temperature of $22 \pm 1^{\circ} \mathrm{C}$. Food and water were available ad libitum. Zeitgeber time (ZT) 0 was defined as lights on, and ZT12 was defined as lights off. Animals were maintained under these conditions for $>2$ weeks before experimental procedures. A separate group of WT and $V i p r 2^{-1-}$ mice, maintained under similar conditions, was used for behavioral assessment. For analyses of wheel-running behavior, mice were transferred to individual cages equipped with running wheels under LD for $>1$ week before transfer to constant darkness (DD). Animals free ran in $\mathrm{DD}$ for at least $10 \mathrm{~d}$ before experimental procedures. Running wheel activity was recorded as the number of wheel revolutions in $10 \mathrm{~min}$ using the Tau program (Mini-Mitter, Sunriver, OR). All scientific procedures were performed in accordance with the United Kingdom Animal (Scientific Procedures) Act 1986.

Slice preparation. Slices were prepared and maintained using methods similar to those described previously (Cutler et al., 2003). Slice preparation was performed during the early subjective day (ZT $1-3$ ), or at the beginning of the animals' inactive phase, with the aid of infrared goggles for animals in DD.

Mice were anesthetized with halothane (AstraZeneca, Macclesfield, UK) followed by cervical dislocation and decapitation. The brain was then removed and placed in $4^{\circ} \mathrm{C}$ artificial CSF (aCSF), $\mathrm{pH} 7.4$, composed of the following (in mM): $124 \mathrm{NaCl}, 2.2 \mathrm{KCl}, 1.2 \mathrm{KH}_{2} \mathrm{PO}_{4}, 2.5 \mathrm{CaCl}_{2}, 1.0$ $\mathrm{MgSO}_{4}, 25.5 \mathrm{NaHCO}_{3}, 10$ D-glucose, and 1.14 ascorbic acid. Coronal brain sections (400 $\mu \mathrm{m}$ thick) were cut using a vibroslicer (Campden Instruments, Leicester, UK) and transferred to an interface style brain slice chamber continuously perfused $(\sim 1.5 \mathrm{ml} / \mathrm{min})$ with oxygenated $\left(\begin{array}{lll}95 \% & \mathrm{O}_{2} / 5 \% & \mathrm{CO}_{2}\end{array}\right)$ aCSF supplemented with $0.0005 \%$ gentamicin (Sigma, Poole, UK) and warmed to $36 \pm 1^{\circ} \mathrm{C}$. Slices were allowed to equilibrate for $>1$ h before electrophysiological recordings.

Electrophysiological recordings. Extracellular multiunit activity (MUA) was recorded from the central SCN for two or more cycles, using glasstipped "suction" electrodes filled with aCSF (supplemental Figs. 1-3, available at www.jneurosci.org as supplemental material). Electrode tips were fabricated from standard borosilicate glass micropipettes with their tips cut down to a diameter of $\sim 100 \mu \mathrm{m}$ using a diamond-tipped pen (Fisher Scientific, Manchester, UK). Pipette tips were subsequently flame polished and bent to an angle of $\sim 45^{\circ}$ over a spirit burner and then attached to a rigid plastic casing, inside which ran an $\mathrm{AgCl}$-coated wire. A syringe was attached to the distal end of the electrode casing, and the whole apparatus was carefully sealed with epoxy resin and flexible rubber tubing. This design allowed electrodes to be positioned over the brain slice using standard micromanipulators such that the tips lay flat over the SCN and could be stabilized by application of negative pressure via the attached syringe. The SCN multiunit signal was differentially amplified $(20,000 \times)$ and bandpass filtered $(300-3000 \mathrm{~Hz})$ via a Neurolog system (Digitimer, Welwyn Garden City, UK) and then digitized $(50,000 \mathrm{~Hz})$ using a micro 1401 mkII interface (Cambridge Electronic Design, Cambridge, UK) and recorded on a personal computer running spike 2 version 5 software (Cambridge Electronic Design).

Drugs. GRP and the $\mathrm{BB}_{2}$ receptor antagonist [D-Phe ${ }^{6}$, Des-Met ${ }^{14}$ ]Bombesin 6-14 Ethylamide (DPDMB) were obtained from Bachem (St. Helens, UK), NMDA was obtained from Sigma, and the $\mathrm{VPAC}_{2}$ antagonist PG 99-465 was a generous gift from Prof. Patrick Robberecht (University of Brussels, Brussels, Belgium). PG 99-465 was stored as a $100 \mu \mathrm{M}$ stock in DMSO and dissolved to the required concentration (10 nM) in
aCSF to give a final DMSO concentration of $0.01 \%$. All other drugs were stored as stock solutions in distilled water. Drug applications were started at ZT 3.5 or 1-2 $\mathrm{h}$ after the start of electrophysiological recordings for animals in DD. For experiments in which GRP and PG 99-465/DPDMB were coapplied, the antagonist was applied 15 min before GRP (i.e., ZT 3.25).

Data analysis. Single-unit activity was discriminated from multiunit recordings off-line (supplemental Fig. $1 a$, available at www.jneurosci.org as supplemental material) with Spike 2 software and scripts designed in-house, using techniques similar to those reported previously (Schaap et al., 2003). Single units were discriminated on the basis of waveform shape and validated as distinct spike shapes by measurement-based clustering using the amplitudes and of dominant positive and negative components (supplemental Fig. $1 j$, available at www.jneurosci.org as supplemental material), their latencies, and maximum slope between the first peak and return to $0 \mathrm{~V}$. Putative single-unit activity was further validated as the contribution of an individual neuron by the presence of a clear refractory period, seen as an epoch in which there were no spikes at the beginning of an interspike interval histogram (supplemental Fig. $1 d, f, h$, available at www.jneurosci.org as supplemental material). For each single unit, the mean waveform of all contributing spikes was calculated for successive $24 \mathrm{~h}$ periods (supplemental Fig. $1 b, c, e, g$, available at www. jneurosci.org as supplemental material). Units were rejected when the average waveform of contributing spikes over successive $24 \mathrm{~h}$ periods showed obvious differences. Using these conservative criteria, we were able to successfully identify two to four single units from each experiment. The total neural activity recorded with a signal-to-noise ratio of $>2: 1$ is reported as MUA (in hertz) (supplemental Fig. 1 $i$, available at www.jneurosci.org as supplemental material). We estimate that this multiunit signal represents the activity of $\sim 10$ SCN neurons, because SCN MUA from WT slices peaked at $43 \pm 5 \mathrm{~Hz}$ and the discharge of single-units peaks at $4.1 \pm 0.3 \mathrm{~Hz}$ (Cutler et al., 2003).

The presence or absence of rhythmicity in neural activity was assessed using curve-fitting software developed in-house. The raw data were transformed by dividing by the value of a line of best fit and then subtracting the mean value of the result. The transformed data were then fitted with a curve of the equation $y=A_{\sin }(B x)$, using the NewtonRaphson iterative method (supplemental Fig. 1i, available at www. jneurosci.org as supplemental material), where $A$ is the amplitude of the rhythm and $B$ is the frequency in radians/h (thus, period in hours = $2 \pi / B)$. The periods of neural rhythms were also estimated by taking the time difference between peak firing on days 1 and 2 in vitro with essentially identical results (supplemental Table 1, available at www. jneurosci.org as supplemental material). A neuron/slice was judged arrhythmic when the best fit curve had 0 amplitude (i.e., a straight line) or had a period of $<12 \mathrm{~h}$ or $>36 \mathrm{~h}$. Neurons/slices were also classified as rhythmic or arrhythmic by three trained experimenters, two of whom were blind to the genotype of the animal that contributed the slice. These ratings agreed with the mathematical analysis in $96 \pm 1 \%$ of cases.

The degree of synchrony among rhythmic SCN neurons was estimated using the time between peak firing of each single unit and the occurrence of the MUA peak from that slice [phase difference (h)], averaged across days 1 and 2 in vitro. Synchrony was also assessed using the width of neural activity peaks, defined as twice the time taken for firing rate to decrease from its daily maximum to $50 \%$ of maximum [peak width (h)], a measure previously shown to indirectly assess synchrony among SCN neurons comprising an MUA recording (Schaap et al., 2003).

Acute drug effects were assessed as the mean single-unit firing rate 10-20 min after the start of drug perfusion compared with the mean discharge in the $10 \mathrm{~min}$ period immediately before drug application.

Data are presented as mean \pm SEM. Proportions of rhythmic neurons were compared by the $\chi^{2}$ test, acute drug-induced changes in firing rate by paired $t$ test, and differences in the periodicity of rhythms by KruskalWallis followed by Dunns test because of their non-Gaussian nature. All other data were analyzed using one-way ANOVA followed by Tukey's test or unpaired $t$ test. Significance was set at $p=0.05$. All statistical tests were performed using GraphPad Prism 3.0 (GraphPad, San Diego, CA).

Animals were classified as rhythmic/arrhythmic on the basis of the last $10 \mathrm{~d}$ wheel running activity in DD by Sokolove-Bushell periodogram 

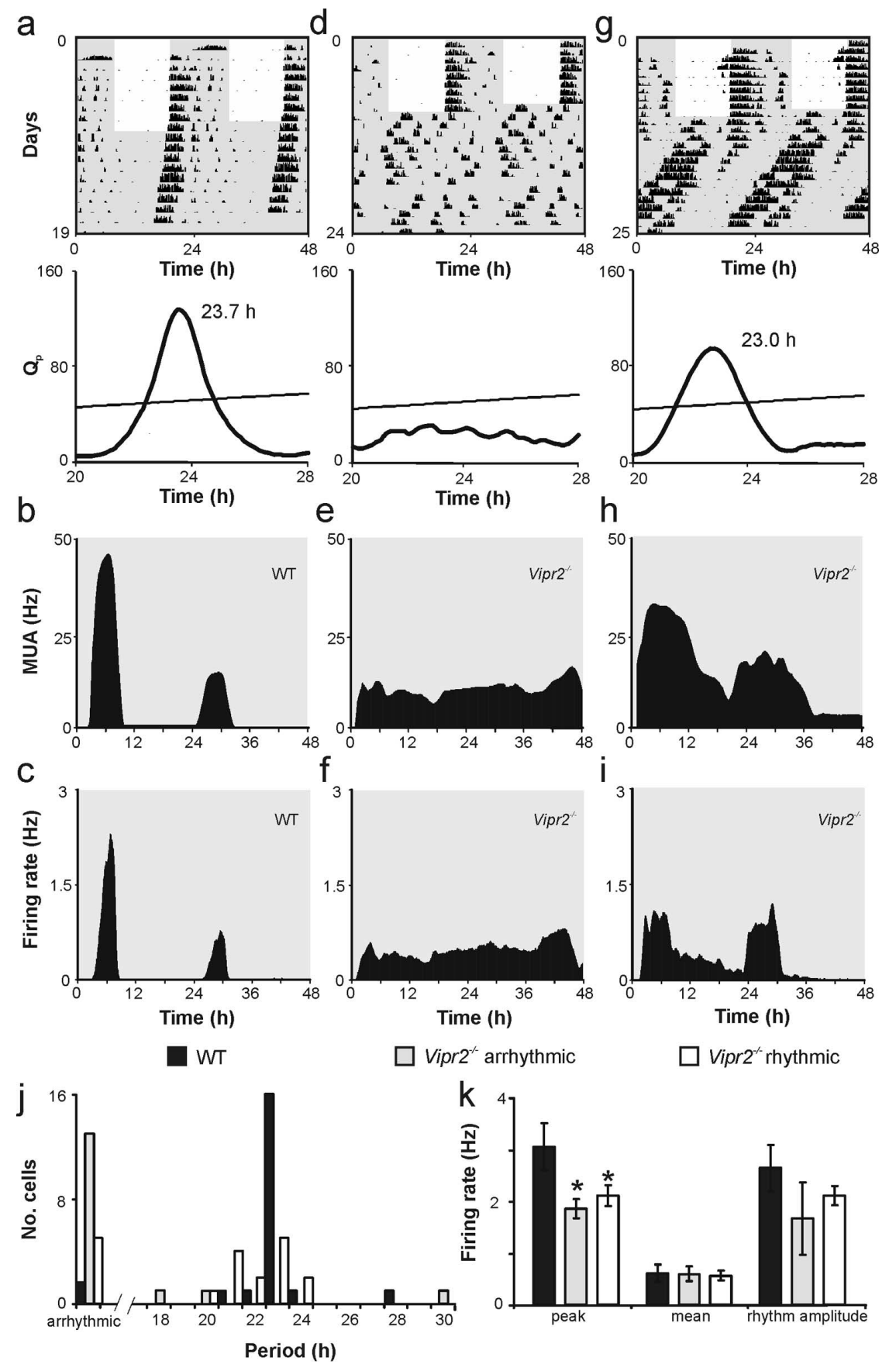

Figure 1. Coherent behavioral rhythms in Vipr2 $2^{-1-}$ mice kept in constant darkness predict the presence of SCN firing rate rhythms. WT mice showed strong rhythms in wheel running $(\boldsymbol{a})$ and multiunit and single-unit electrical activity $(\boldsymbol{b}, \boldsymbol{c})$ with estimated periods close to $24 \mathrm{~h}(\boldsymbol{j})$. In slices from all behaviorally arrhythmic Vipr $2^{-1-}$ mice (d), MUA (e) and most single units are also arrhythmic $(\boldsymbol{f}, \boldsymbol{j})$. However, some Vipr ${ }^{-/-}$mice expressed short-period rhythms in behavior $(\boldsymbol{g})$, and in SCN slices from these mice, MUA and the discharge of most single units displayed similar short-period rhythmicity $(\boldsymbol{h}, \boldsymbol{i}, \boldsymbol{j})$. The peak, but not mean, firing rates of SCN neurons from both rhythmic and arrhythmic Vipr $2^{-1-}$ mice were significantly lower than those from WT animals ( $\boldsymbol{k}$; Tukey's test). ${ }^{*} p<0.05$ versus WT. MUA and single-unit data are plotted as mean firing rate (in hertz) every minute. Data in $\boldsymbol{k}$ correspond to average single-unit values \pm SEM.

(Sokolove and Bushell, 1978) using analysis software developed inhouse. Rhythmic components were deemed significant when they crossed a line corresponding to $p<0.001$.

\section{Results}

Behavioral rhythmicity predicts the proportion of oscillating Vipr $2^{-1-}$ SCN neurons

In cell culture, a minority of neonatal Vipr $2^{-1-}$ SCN neurons express circadian rhythms (Aton et al., 2005); however, despite demonstrations that some Vipr2 $2^{-1-}$ mice express rhythmic wheel-running behavior (Hughes et al., 2004), electrical/molecular oscillations have not yet been observed in the SCN of adult animals (Harmar et al., 2002; Cutler et al., 2003). Here, we developed a sensitive in vitro electrophysiological recording technique, which simultaneously assesses MUA and single-unit discharge, to relate the electrical activity of adult Vipr2 ${ }^{-1-}$ SCN neurons to the animals' behavioral phenotype.

All WT mice examined in DD $(n=8)$ showed clear wheel-running rhythms with a mean period of $23.8 \pm 0.1 \mathrm{~h}$ (Fig. 1a), and SCN slices from these animals expressed MUA oscillations with similar periods (Fig. 1b) (estimated period, $23.8 \pm$ $0.3 \mathrm{~h}$ ). In addition, of the 22 individual neurons (Fig. $1 c, j$ ) we could reliably discriminate from these MUA recordings, 20 ( $\sim 91 \%)$ displayed high-amplitude $(2.8 \pm$ $0.5 \mathrm{~Hz}$ ) firing rate rhythms. Similarly, in all ( six of six) slices prepared from behaviorally rhythmic Vipr $2^{-/-}$mice (Fig. $1 g$ ), we recorded rhythms in the SCN MUA (Fig. $1 h$ ) with estimated periods close to the measured behavioral rhythm (slices, $21.6 \pm 1.2 \mathrm{~h}$; behavior, $22.2 \pm 0.5 \mathrm{~h}$ ). Again, a high proportion of the individual SCN neurons detected (16 of $21 ; \sim 76 \%$ ) (Fig. $1 i, j$ ) from these rhythmic Vipr $2^{-/-}$ mice showed rhythmic electrical activity with similar estimated periods to MUA and behavior. In contrast, we did not observe MUA rhythms in any SCN slices prepared from behaviorally arrhythmic Vipr $2^{-/-}$mice (Fig. 1d,e) $(n=6)$ and a significantly smaller proportion of cells detected in these slices expressed coherent circadian oscillations in firing rate ( 3 of 16 ; $\sim 19 \%$ ) (Fig. 1j) ( $\chi^{2}$ test, arrhythmic vs rhythmic Vipr2 ${ }^{-/-}$mice; $p<0.05$ ).

Previous work suggests that adult Vipr2 ${ }^{-1-}$ SCN neurons show decreased peak firing rates compared with WT (Cutler et al., 2003), whereas the genotypes' average firing rates do not differ in neonatal cultures (Aton et al., 2005). Our data support both observations indicating that the peak firing rates of detected Vipr $2^{-/-}$neurons were significantly lower than WT, whereas the mean daily firing rates were not (Fig. $1 k$ ). Thus, these results indicate a reduced ability of Vipr $2^{-1-}$ SCN neurons to "gate" their activity so that high discharge occurs only at specific points in the circadian cycle. Accordingly, the amplitude of oscillating cells in SCN slices from behaviorally rhythmic Vipr $2^{-/-}$mice was slightly, but not significantly, lower than WT (Fig. $1 k$ ).

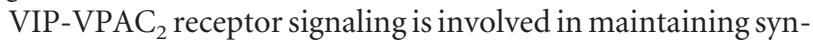
chrony among neonatal SCN neurons in high-density culture (Aton et al., 2005). Because MUA rhythms were not detected in 
recordings from behaviorally arrhythmic Vipr2 $2^{-1-}$ mice, we are unable to effectively assess synchrony within such slices. However, we observed robust SCN MUA rhythms in recordings from behaviorally rhythmic Vipr $2^{-1-}$ mice, and here the phase difference between rhythmic single units and the MUA rhythm was not significantly different from that seen in WT slices (phase difference, $1.1 \pm 0.2 \mathrm{~h}$ vs $0.7 \pm 0.1 \mathrm{~h}$, respectively; $t$ test, $p>0.05$ ). Although some SCN neurons express circadian rhythms in antiphase to the population (Quintero et al., 2003; Yamaguchi et al., 2003), we did not observe any cells $>3 \mathrm{~h}$ out of phase with the MUA rhythm. This likely reflects the fact that our MUA signal represents a sample from a small group of neighboring cells, since phase difference among SCN neurons is believed to be partially dependent on interneuronal distance (Schaap et al., 2003). In addition, the widths of multiunit or single-unit peaks observed in our recordings (an indirect measure of synchrony) were similar to those reported previously for single/small clusters of rat SCN neurons (Schaap et al., 2003) and were not significantly different between rhythmic Vipr $2^{-1-}$ or WT SCN slices (supplemental Table 1, available at www.jneurosci.org as supplemental material). Thus, in adult Vipr $2^{-/}$animals expressing behavioral rhythms, we found that for SCN neurons in close proximity, there is no evidence that the $\mathrm{VPAC}_{2}$ receptor is required for cellular synchrony in the SCN.

In summary, we established a correlation between the presence or absence of behavioral rhythmicity in Vipr $2^{-/-}$mice and the percentage of rhythmic neurons detected in their SCN. These within-genotype differences suggest that unknown factors compensate for the loss of the $\mathrm{VPAC}_{2}$ receptor in some animals and could indicate that the locomotor rhythms observed in a subset of Vipr $2^{-1-}$ mice are SCNdriven.

\section{Exogenous GRP promotes cellular rhythmicity in the Vipr2 $^{-1-} \mathrm{SCN}$}

Because the SCN in some Vipr $2^{-/-}$mice continue to oscillate, we hypothesized that other neurochemical mechanisms function to promote SCN cellular rhythmicity in these individuals and enable the expression of coherent behavioral rhythms. Because of its functional similarities and partial colocalization with VIP (see Introduction), we tested whether GRP could promote neuronal rhythms in the SCN of Vipr $2^{-1-}$ mice.

Initially, we examined the effects of $48 \mathrm{~h}$ GRP applications (50 $\mathrm{nM}$ ) on SCN slices prepared from behaviorally unscreened Vipr $2^{-1-}$ mice, taken from LD conditions. As predicted from previous data (Hughes et al., 2004) and our experiments on behaviorally phenotyped animals, six of nine control Vipr $2^{-/-} \mathrm{SCN}$ slices lacked MUA rhythms (Fig. 2a), whereas the remaining three exhibited weak oscillations. In addition, of the 30 single units discriminated from these MUA recordings (Fig. $2 b$ ) only nine $(30 \%)$ expressed detectable circadian rhythms (estimated period, $22.2 \pm 1.2 \mathrm{~h}$ ), a similar proportion to that observed in neonatal SCN cultures derived from multiple Vipr $2^{-/-}$animals (Aton et al., 2005).

In contrast, three of four SCN slices perfused with GRP displayed overt MUA rhythms (Fig. 2c) and, within these slices, a significantly greater proportion of discriminated cells oscillated (Fig. $2 d, i$ ) ( 11 of $15 ; \sim 73 \% ; \chi^{2}$ test; $p<0.05$ vs untreated slices; estimated period, $22.2 \pm 1.3 \mathrm{~h}$ ). Furthermore, single-unit rhythms in these GRP-treated Vipr $2^{-/-}$slices were of significantly greater amplitude than in untreated slices (Fig. 2k) (Tukey's test; $p>0.05$ ).

These observations demonstrate that, in the absence of VIP$\mathrm{VPAC}_{2}$ receptor signaling, GRP promotes SCN cellular rhyth- micity. Additional experiments indicated that continual perfusion with GRP was necessary to observe this effect, because slices given shorter $(24 \mathrm{~h})$ applications of GRP did not maintain the induced rhythm beyond the duration of application (data not shown). These actions of GRP were mediated specifically by the $\mathrm{BB}_{2}$ receptor because, when GRP was coapplied with its receptor antagonist DPDMB (100 nM), no slices tested displayed SCN MUA rhythms (Fig. $2 e)(n=4)$, and only 1 of 12 cells examined displayed obvious rhythmic discharge.

To rule out the possibility that GRP promoted SCN rhythms in Vipr $2^{-/-}$slices by some generalized excitatory action, we tested whether another excitatory agent, NMDA, produced similar effects. Rhythms in SCN MUA were not observed in any Vipr $2^{-1-}$ slices chronically perfused with NMDA $(20 \mu \mathrm{M} ; n=4)$ (Fig. $2 g$ ). Indeed, the majority ( 9 of 12) of SCN neurons recorded in these Vipr $2^{-/-}$slices failed to display rhythmic electrical discharge (Fig. $2 h, i$ ). In addition, although both GRP and NMDA acutely elevated single-unit discharge (Fig. 2j) (paired $t$ test; $p<$ 0.05 compared with predrug values), unlike GRP, NMDA did not increase the amplitude of oscillation in rhythmic neurons (Fig. $2 k$ ). Thus, facilitation of Vipr $2^{-1-} \mathrm{SCN}$ rhythmicity by exogenous GRP cannot be mimicked by another excitatory agent that does not signal via the $\mathrm{BB}_{2}$ receptor.

\section{$\mathrm{BB}_{2}$ receptor signaling enables the expression of cellular rhythms in the Vipr2 ${ }^{-/-} \mathrm{SCN}$}

Because exogenous GRP appeared to promote cellular rhythmicity in the Vipr $2^{-/-}$SCN, we next investigated whether oscillating neurons detected in the SCN of behaviorally rhythmic individuals use $\mathrm{BB}_{2}$ receptor signaling to sustain rhythms.

Under control conditions, all SCN slices from Vipr ${ }^{-1-}$ mice that were behaviorally rhythmic in DD displayed clear MUA rhythms (see above). However, when SCN slices from behaviorally rhythmic Vipr $2^{-/-}$mice (Fig. $3 a, d$ ) were chronically perfused with the $\mathrm{BB}_{2}$ receptor antagonist DPDMB, electrical activity was reversibly suppressed, and four of six failed to display MUA rhythms (Fig. 3b) (supplemental Fig. 2, available at www. jneurosci.org as supplemental material). Most individual SCN neurons in these four arrhythmic slices (11 of 12) also lacked detectable rhythms (Fig. 3c). However, two slices maintained high-amplitude MUA rhythms in the presence of DPDMB (Fig. $3 e$ ), suggesting that factors other than $\mathrm{GRP}-\mathrm{BB}_{2}$ receptor signaling contribute to the maintenance of SCN rhythms in a subset of Vipr $2^{-/-}$mice. Overall, only $\sim 31 \%$ of discriminated SCN neurons (6 of 19) from behaviorally rhythmic Vipr $2^{-/-}$mice sustained firing rate rhythms in the presence of DPDMB (Fig. $3 f$ ), significantly less than the proportion of oscillating cells $(\sim 76 \%)$ seen in untreated slices $\left(\chi^{2}\right.$ test; $\left.p<0.05\right)$. The amplitude of the rhythm in these cells $(1.6 \pm 0.2 \mathrm{~Hz})$ tended to be lower than that seen in control slices from behaviorally rhythmic Vipr $2^{-1-}$ mice and was significantly lower than that in WT neurons (Tukey's test; $p<0.05$ ).

We next tested whether exogenous GRP could promote neuronal rhythms in SCN slices from Vipr $2^{-/-}$mice that did not express coherent behavioral rhythms under DD (Fig. 3g,j). No slices from these arrhythmic mice expressed circadian MUA rhythms under control conditions, whereas in agreement with our data from LD animals, three of four slices displayed highamplitude MUA oscillations in the presence of GRP (Fig. 3h). In these slices, the majority of discriminated individual SCN neurons ( 7 of $12 ; \sim 58 \%$ ) (Fig. $3 i$ ) showed high-amplitude $(2.3 \pm 0.5$ $\mathrm{Hz}$ ) firing rate rhythms (estimated period, $22.0 \pm 0.6 \mathrm{~h}$ ), although some cells displayed very fast oscillations outside the circadian range (Fig. 3l). The proportion of rhythmic neurons in 

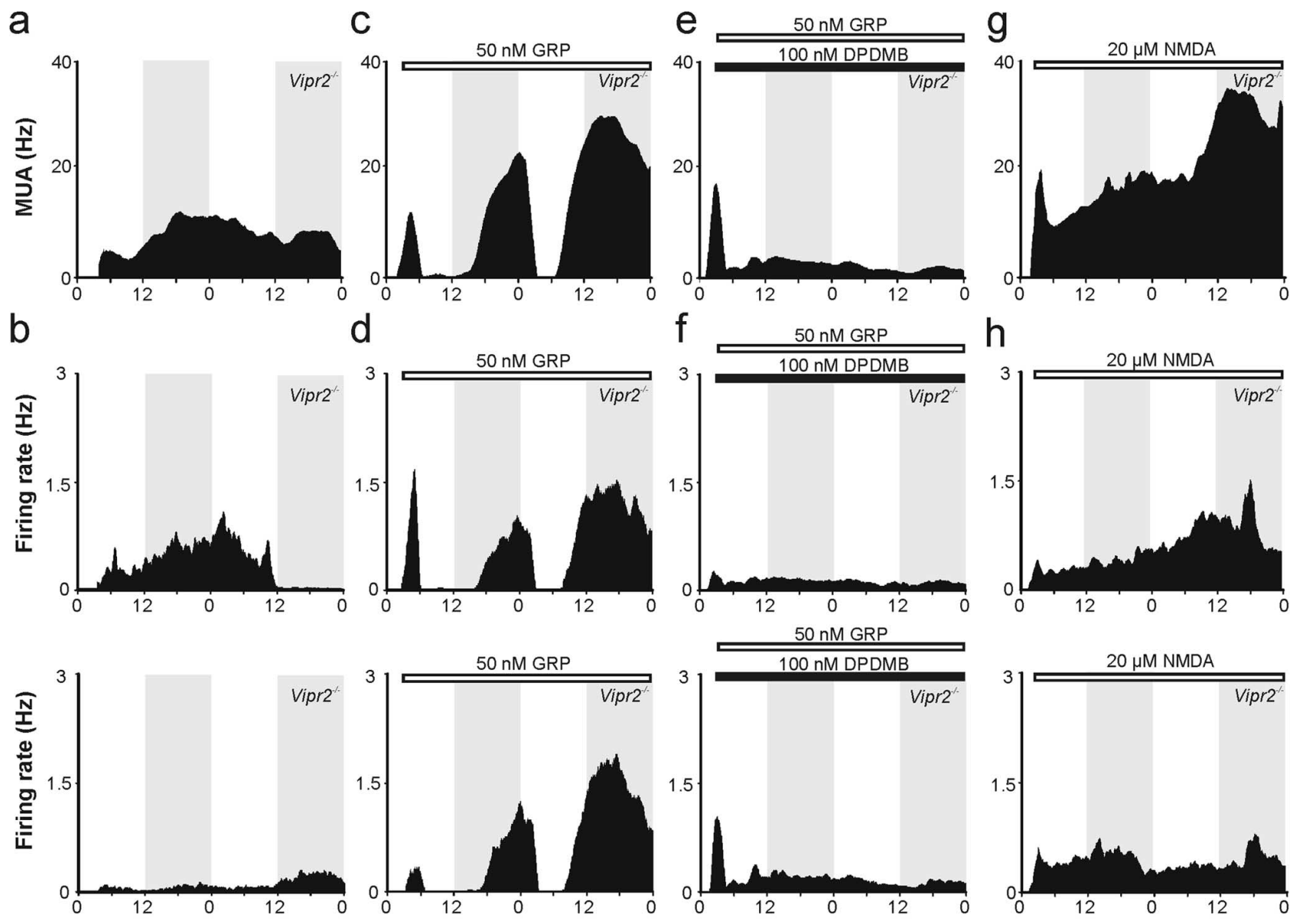

Projected Zeitgeber time (h) Projected Zeitgeber time (h)

Projected Zeitgeber time (h)
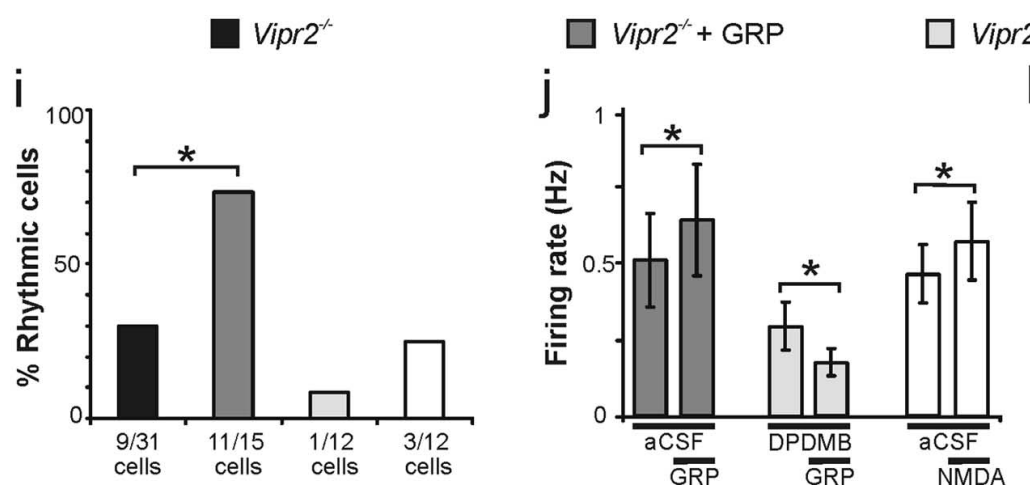

pr $2^{-\alpha}+$ DPDMB/GRP

$\mathrm{k}$

Projected Zeitgeber time (h)

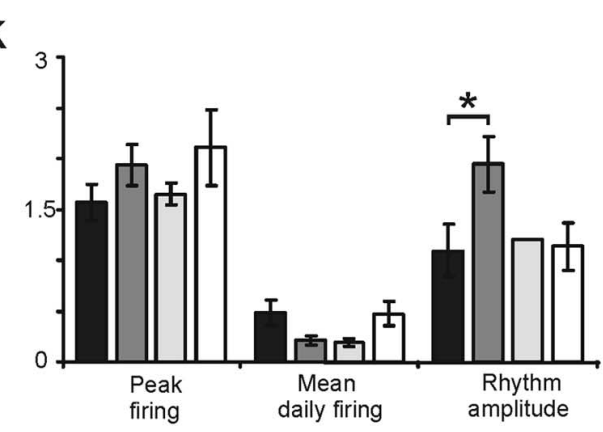

Figure 2. GRP promotes cellular rhythmicity in the Vipr $2^{-/}$SCN. The majority of slices prepared from Vipr $2^{-/-}$mice housed under LD (6 of 9$)$ lack rhythms in MUA (a), and most single units detected within these slices are also arrhythmic $(\boldsymbol{b}, \boldsymbol{i})$. Chronic application of GRP to Vipr $2^{-1-} \mathrm{SCN}$ slices promoted MUA rhythms $(\boldsymbol{c})$ and robust, short-period rhythmicity in most single units $(\boldsymbol{d}$, $l$; $\chi^{2}$ test). When applied in the presence of the $\mathrm{BB}_{2}$ receptor antagonist DPDMB, GRP failed to promote rhythms in SCN multiunit $(\boldsymbol{e})$ or single-unit $(\boldsymbol{f}, \boldsymbol{i})$ activity. Another excitatory agent, NMDA, did not promote MUA $(\boldsymbol{g})$ or single-unit electrical rhythms $(\boldsymbol{h}, \boldsymbol{i})$ in SCN slices from Vipr $2^{-1-}$ mice. Ten to 20 min after application of GRP or NMDA, the single-unit firing rate (mean \pm SEM) was significantly increased compared with $10 \mathrm{~min}$ before drug application, whereas firing rates were significantly decreased in the same time period after GRP application in the presence of DPDMB $(j$; paired $t$ tests). Whereas none of the drug treatments significantly altered peak or mean daily firing rates ( $\boldsymbol{k}$; Tukey's test; $p>0.05)$, GRP treatment increased the amplitude of oscillating cells compared with control Vipr ${ }^{-1-}$ SCN neurons $\left(\boldsymbol{k} ; t\right.$ test). ${ }^{*} p<0.05$. Shaded areas in $\boldsymbol{a}-\boldsymbol{h}$ correspond to periods of darkness extrapolated from the previous $L D$ cycle. The bars in $\boldsymbol{c}-\boldsymbol{h}$ indicate timing and duration of drug application. Error bars in $\boldsymbol{j}$ and $\boldsymbol{k}$ correspond to \pm SEM.

these GRP-treated slices was significantly greater than in untreated SCN slices from arrhythmic Vipr2 ${ }^{-1-}$ mice $\left(\chi^{2}\right.$ test; $p<$ $0.05)$. Preliminary experiments indicate that phasic application of $50 \mathrm{~nm}$ GRP (6 h on, $18 \mathrm{~h}$ off for three cycles) also promotes cellular rhythmicity in SCN slices from arrhythmic Vipr2 ${ }^{-/-}$ mice (supplemental Fig. 2, available at www.jneurosci.org as supplemental material). During these phasic GRP treatments, increases in electrical activity appeared to precede peptide applica- tion, consistent with the accelerated cellular rhythms observed in the SCN of Vipr2 ${ }^{-/-}$mice.

GRP promotes cellular rhythmicity in the WT SCN in the absence of $\mathrm{VPAC}_{2}$ receptor signaling

A previous study suggests that the $\mathrm{BB}_{2}$ receptor is not essential for SCN pacemaker function (Aida et al., 2002). Because our data indicate that GRP promotes cellular rhythmicity in the Vipr2 ${ }^{-/-}$ 

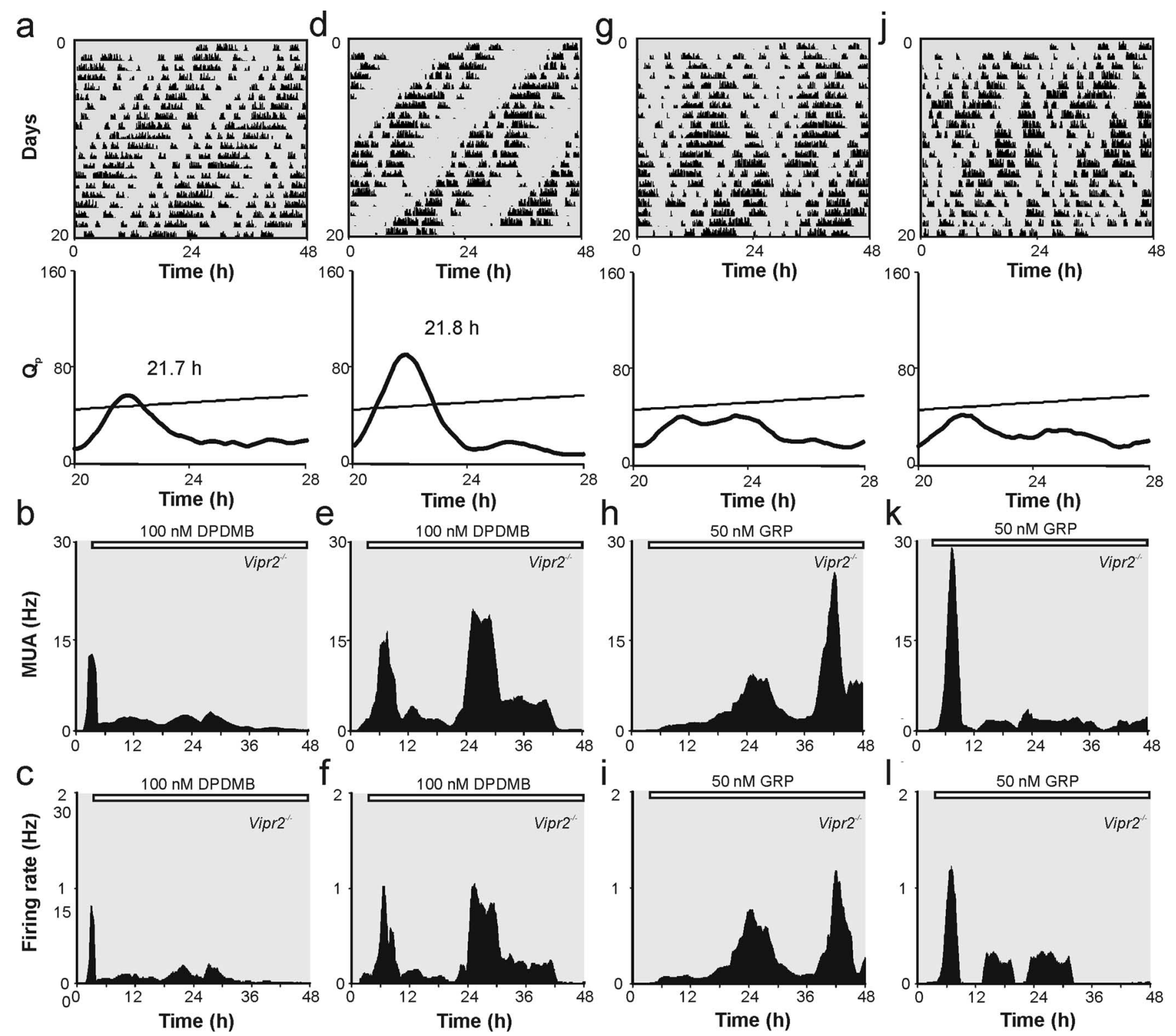

Figure 3. $G R P-B B_{2}$ receptor signaling partially accounts for $\mathrm{SCN}$ electrical activity rhythms in Vipr $2^{-/-}$mice. Long-term perfusion of brain slices from behaviorally rhythmic Vipr $2^{-/-}$mice $(\boldsymbol{a}$, $\boldsymbol{d}$ ) with the $\mathrm{BB}_{2}$ receptor antagonist DPDMB prevented MUA rhythms in four to six slices $(\boldsymbol{b})$ and rendered the majority of single units arrhythmic (c). However, some antagonist-treated slices showed clear circadian rhythms in multiunit and single-unit discharge $(\boldsymbol{e}, \boldsymbol{f})$. Conversely, in the presence of GRP, robust MUA rhythms were observed in three of four SCN slices $(\boldsymbol{h})$ from behaviorally arrhythmic Vipr2 $2^{-/-}$mice $(\boldsymbol{g}, \boldsymbol{j})$ and the majority of single units detected $(\boldsymbol{i})$. GRP failed to induce MUA rhythms in one slice $(\boldsymbol{k})$ but caused short-period oscillations (outside the circadian scale) in single-unit discharge $(\boldsymbol{I})$. The bars in $\boldsymbol{b}$-I indicate timing and duration of drug application.

SCN, we investigated whether GRP was involved in the maintenance of cellular rhythms in the SCN of WT animals.

We recorded SCN MUA in nine control WT slices prepared from animals housed under LD. Consistent with previous studies (Bouskila and Dudek, 1993; Gribkoff et al., 1998; Mrugala et al., 2000), all WT slices showed clear circadian rhythms in SCN MUA (Fig. 4a) (supplemental Figs. 1, 3, available at www.jneurosci.org as supplemental material) (estimated period, $23.7 \pm 0.4 \mathrm{~h}$ ). As observed in WT slices from DD animals, the majority of SCN neurons discriminated from these MUA recordings (27 of 30; $\sim 90 \%$ ) showed high-amplitude oscillations with similar estimated periods (Fig. $4 b, f$ ).

Similarly, when chronically perfused with the $\mathrm{BB}_{2}$ receptor antagonist DPDMB (100 nM), all WT slices showed MUA rhythms (Fig. $4 b)(n=4)$, as did the majority (12 of $14 ; \sim 86 \%)$ of individual SCN neurons (Fig. $4 c, f$ ). Neither the peak and mean daily firing rates nor rhythm amplitude in DPDMB-treated WT SCN neurons differed significantly from untreated WT cells (Fig. $4 g)$. Therefore, in agreement with the previous demonstration of robust behavioral rhythmicity in mice lacking the $\mathrm{BB}_{2}$ receptor (Aida et al., 2002), these data indicate that signaling via this receptor is not essential for neural rhythms in the WT SCN.

We next investigated whether application of exogenous GRP altered electrical oscillations in the WT SCN. When constantly perfused with GRP (50 nM), three of four WT slices maintained MUA rhythms (Fig. 4d), and one slice became arrhythmic. GRP treatment did not alter the peak and mean daily firing rate or rhythm amplitude in WT SCN neurons (Fig. $4 f$ ), but the proportion showing clear firing rate rhythms (Fig. $4 e, f$ ) was significantly reduced compared with untreated slices ( 8 of $13 ; \sim 62 \%$; $\chi^{2}$ test; 
a

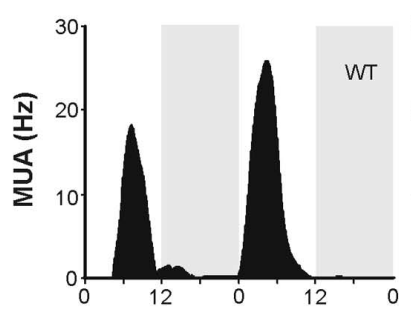

b
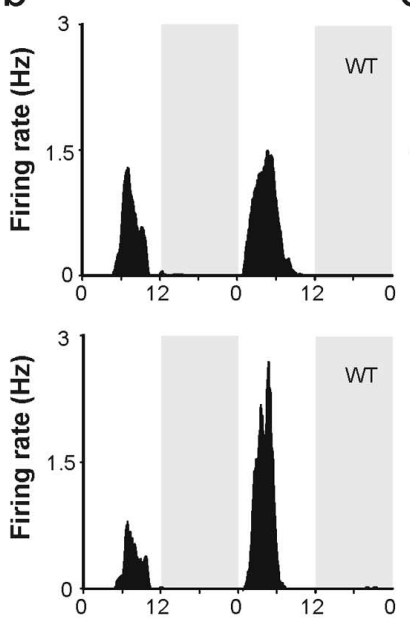

Projected Zeitgeber time (h)

WT

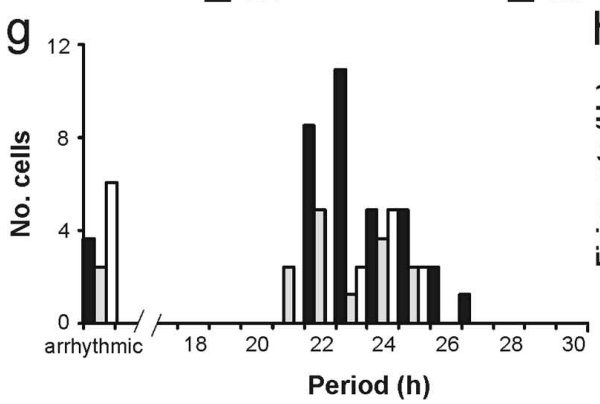

Period (h)
C
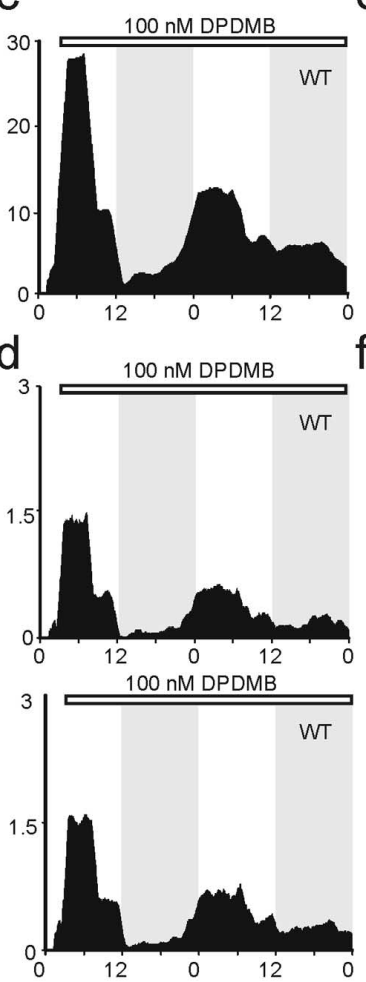

WT

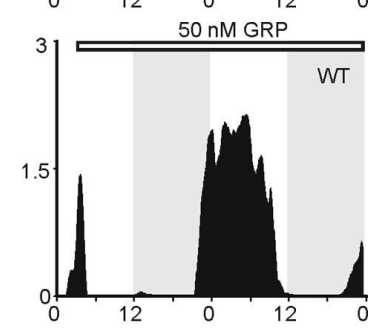

Projected Zeitgeber time (h)

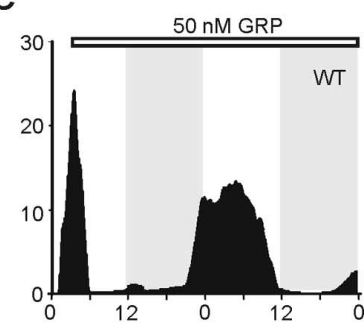

$f$

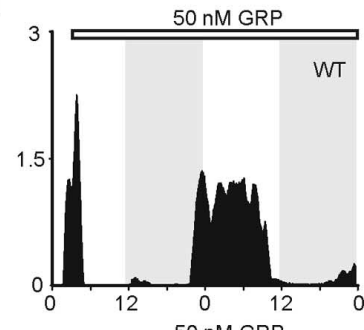

WT

(a)
oscillated with greater amplitude than those in PG 99-465-only-treated slices (Fig. 5f) ( $t$ test; $p<0.01)$. Interestingly, the estimated period of cellular rhythms observed in slices cotreated with PG 99645 and GRP $(22.1 \pm 0.3 \mathrm{~h})$ was significantly shorter than that observed in untreated WT slices (Dunns test; $p<0.05$ ) and essentially identical to that observed in Vipr2 ${ }^{-/-}$slices/animals. Therefore, these data indicate that the ability of GRP to promote cellular rhythmicity in the Vipr $2^{-1-} \mathrm{SCN}$ is not attributable to an as yet uncharacterized developmental abnormality. In addition, our findings highlight a potential new role for GRP in the regulation of rhythmicity in the WT SCN, possibly as one of multiple intercellular signaling systems that enable the SCN to express a coherent circadian output.

Figure 4. GRP-BB receptor signaling is not essential for circadian rhythms in WT SCN electrical activity. SCN slices prepared from WT mice housed under LD displayed robust rhythms in MUA $(\boldsymbol{a})$ and single-unit discharge $(\boldsymbol{b}, \boldsymbol{g})$. Chronic application of the $\mathrm{BB}_{2}$ receptor antagonist DPDMB (100 nM) to WT slices did not prevent multiunit or single-unit firing rate rhythms $(\boldsymbol{c}, \boldsymbol{d}, \boldsymbol{g})$. The

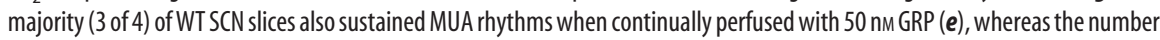
(No.) of single units showing circadian firing rate rhythms $(\boldsymbol{f})$ was reduced $(\boldsymbol{g})$. Neither peak/mean daily firing rates nor the amplitude of rhythmic cells was significantly altered by GRP or DPDMB ( $\boldsymbol{h}$; Tukey's test; $\boldsymbol{p}>0.05$ ). Shaded areas in $\boldsymbol{a}-\boldsymbol{f}$ correspond to periods of darkness extrapolated from the previous $L D$ cycle. The bars in $\mathbf{c}-\boldsymbol{f}$ indicate timing and duration of drug application. Error bars in $\boldsymbol{h}$ correspond to \pm SEM.

$p<0.05)$. Surprisingly, therefore, whereas GRP- $\mathrm{BB}_{2}$ receptor signaling does not appear necessary for normal rhythms in SCN electrical activity, sustained GRP receptor stimulation may be detrimental to the expression of neural rhythms by these structures.

In contrast to the effects of $\mathrm{BB}_{2}$ receptor blockade, when WT slices were chronically treated with a $\mathrm{VPAC}_{2}$ receptor antagonist (10 nм PG 99-465) (Moreno et al., 2000), MUA was suppressed (Fig. $5 a$ ) for the duration of application ( $48 \mathrm{~h}$ ), such that only one of five slices sustained MUA rhythms. In addition, only $27 \%$ (4 of 15) of the individual SCN neurons examined expressed firing rate rhythms in the presence of the antagonist (Fig. $5 b, e$ ). In agreement with previous studies (Cutler et al., 2003), SCN neurons in PG 99-465-treated slices exhibited decreased peak firing

\section{Discussion}

Using a novel, continuous electrophysiological recording system, we now establish that rhythmic neuronal activity is present in the adult Vipr2 $2^{-1-} \mathrm{SCN}$ in vitro. We reveal a striking correlation between the expression of circadian oscillations in SCN single-unit and multiunit activity and behavioral rhythmicity in Vipr2 ${ }^{-/-}$mice, raising the possibility that the SCN clock of these mice retains enough function to drive behavioral rhythms. In support of this suggestion, the estimated periods of SCN neuronal rhythms from this group of mutant mice closely corresponded to those of the animals' behavioral activity.

Interestingly, cellular rhythmicity in Vipr2 ${ }^{-1-}$ mice appears partially dependent on $\mathrm{BB}_{2}$ receptor signaling, whereas in WT mice, it is not. In addition, exogenous GRP promotes the expression of single and multiunit electrical oscillations in SCN slices from behaviorally arrhythmic Vipr2 ${ }^{-/-}$mice but appears detrimental to rhythms in the WT SCN. However, GRP enables WT SCN neurons to sustain rhythms when $\mathrm{VPAC}_{2}$ receptors are 

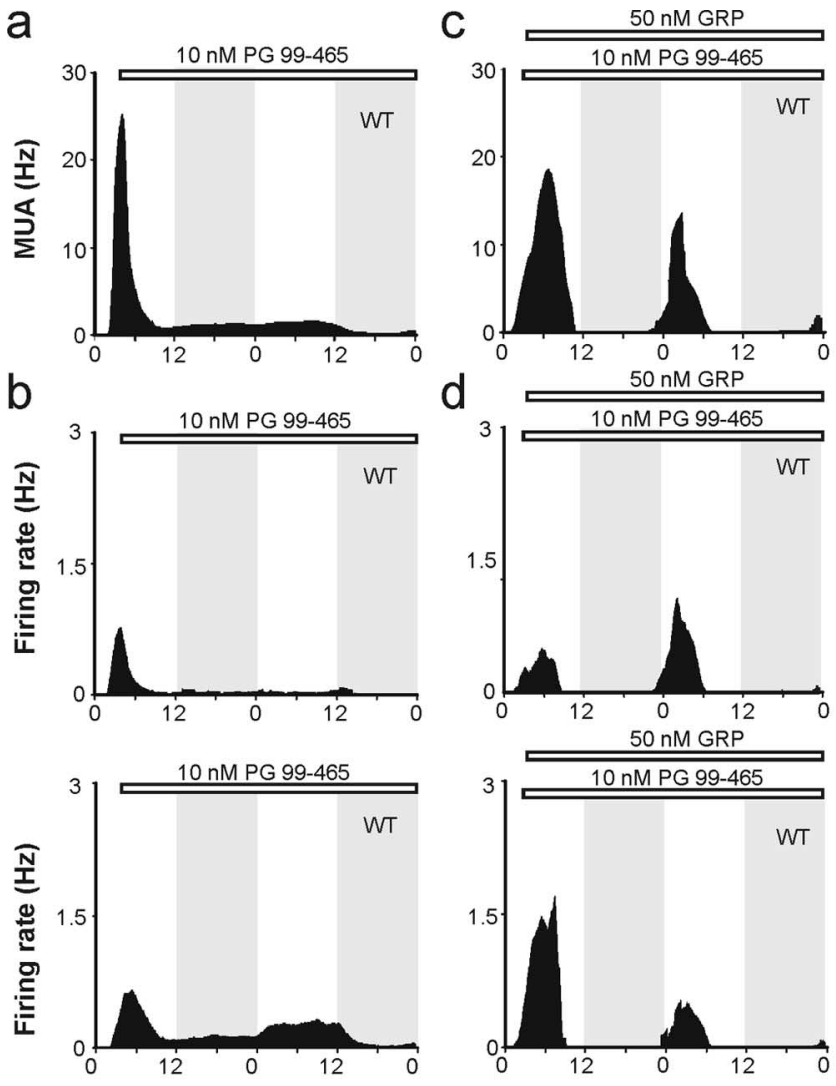

Projected Zeitgeber time (h)

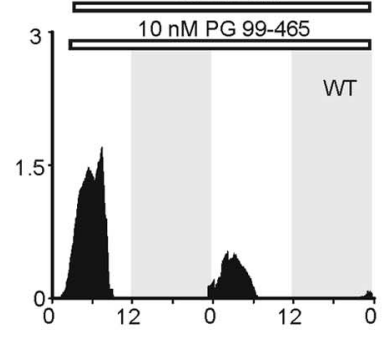

Projected Zeitgeber time (h)

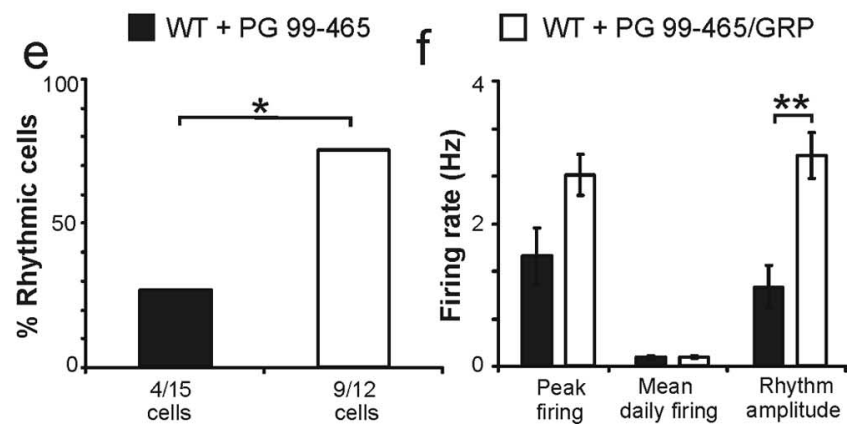

Figure 5. GRP promotes cellular rhythmicity in the WTSCN in the absence of VPAC 2 receptor signaling. Long-term perfusion of WT slices with the $\mathrm{VPC}_{2}$ receptor antagonist $\mathrm{PG}$ 99-465 abolishes circadian rhythms in MUA $(\boldsymbol{a})$ and most single units $(\boldsymbol{b}$, top, $\boldsymbol{e})$, although some neurons expressed low-amplitude rhythms ( $\boldsymbol{b}$, bottom). Chronic application of GRP promoted MUA rhythms in VPAC, receptor antagonist-treated WT SCN slices $(\boldsymbol{c})$ and significantly increased the number of rhythmic neurons detected $\left(\boldsymbol{d}, \boldsymbol{e} ; \chi^{2}\right.$ test). Single units oscillated with higher amplitude in the presence of GRP compared with PG 99-465-only-treated slices ( $\boldsymbol{f} ; t$ test). ${ }^{*} p<0.05$; ${ }^{* *} p<0.01$. The shaded areas in $\boldsymbol{a}-\boldsymbol{d}$ correspond to periods of darkness extrapolated from the previous $L D$ cycle. The bars indicate timing and duration of drug application. Error bars in $\boldsymbol{f}$ correspond to \pm SEM.

pharmacologically blocked, a treatment that, on its own, dramatically reduces the expression of cellular oscillations. Previous studies implicated GRP in photic entrainment (Piggins et al., 1995; Romijn et al., 1996; Aida et al., 2002; Antle et al., 2005), but the present data raise the possibility that endogenous GRP-BB signaling contributes to sustaining neuronal rhythms in the SCN. In this schema, GRP and other signaling factors and pathways (see below) are elements in a network of partially redundant systems regulating SCN rhythmicity and in which VIP-VPAC 2 signaling is a key component.
Our findings broadly agree with the recent observation that $\sim 30 \%$ of cultured neonatal SCN neurons sustain electrical rhythms in the absence of VIP-VPAC 2 signaling (Aton et al., 2005 ) but extend them to adult neurons in an intact slice preparation. In this previous study, it was not possible to correlate the proportion of rhythmic neurons with an individual animals' behavior, because SCN cultures were derived from several mice. Our data suggest that some animals may have contributed significantly more rhythmic neurons to the culture than others. In addition, there are a number of differences in the SCN electrical activity of adult Vipr $2^{-1-}$ slices and cultured neonatal neurons. Whereas neonatal Vipr2 ${ }^{-/-}$SCN neurons do not differ from WT with regard to mean period or firing rate (Aton et al., 2005), we observed reduced peak firing rates, rhythm amplitude, and shorter estimated periods in adult Vipr $2^{-1-}$ SCN cells. Such discrepancies may relate to the age of the cells under study or to differences in the network architecture between culture and slice.

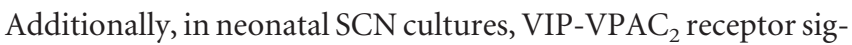
naling is necessary for synchrony among rhythmic neurons (Aton et al., 2005), whereas in SCN slices from behaviorally rhythmic Vipr $2^{-/-}$mice, we found no evidence of asynchrony among small populations of cells. It is possible that the same mechanisms that enable this subset of Vipr $2^{-/-}$mice to sustain circadian rhythms also promote cellular synchrony.

It is notable that when overt behavioral rhythms are detected in mouse models with impaired VIP-VPAC 2 receptor signaling, endogenous periods are shortened with respect to WT mice (Colwell et al., 2003; Hughes et al., 2004; Aton et al., 2005). Intriguingly, a similar spectrum of circadian dysfunction is observed in flies lacking the key clock neuropeptide, pigment dispersing factor, with most individuals rendered arrhythmic and a small proportion maintaining behavioral rhythms with shortened periods (Renn et al., 1999; Peng et al., 2003; Lin et al., 2004). Presumably, the mechanisms that enable sufficient organization of the circadian pacemaker to drive behavioral rhythms in the absence of key signaling peptides are not capable of sustaining near $24 \mathrm{~h}$ clock activity. In support of this suggestion, rhythmic neurons recorded from GRP-treated SCN slices lacking functional $\mathrm{VPAC}_{2}$ receptors appeared to oscillate with shorter periods than those estimated for control WT cells.

The reduced peak firing rates and smaller proportion of rhythmic SCN neurons from adult Vipr $2^{-/-}$and $\mathrm{VPAC}_{2}$ receptor antagonist-treated WT slices (Cutler et al., 2003; ou study) suggest that VIP-VPAC 2 receptor signaling is important for cells to gate their electrical activity. Activation of the $\mathrm{BB}_{2}$ receptor produces primarily excitatory responses in cells throughout the rodent SCN (Piggins and Rusak, 1993; Piggins et al., 1994, 2005; Reynolds and Pinnock, 1997; Cutler et al., 2003; our study) and, like VIP, GRP is believed to be important for communicating phase information between neurons within the circadian clock (Antle et al., 2005). Interestingly, GRP, which promotes cellular rhythmicity in the Vipr $2^{-1-} \mathrm{SCN}$, also increases the amplitude of such rhythms compared with those observed in untreated slices, supporting previous suggestions that a threshold of neuronal activity is required to sustain clock function (Van den Pol and Obrietan, 2002; Nitabach et al., 2002; Yamaguchi et al., 2003; Lundkvist et al., 2005). Surprisingly, another agent that excites the majority of SCN neurons, NMDA (Schmahl and Bohmer, 1997; Cutler et al., 2003), does not increase the amplitude or proportion of cells expressing rhythms in the Vipr2 ${ }^{-/-}$SCN. Therefore, the mechanism by which GRP promotes neural rhythms in the SCN of Vipr $2^{-/-}$is not simply explained by an increase in cellular activity. 
Our observation that exogenous GRP enables WT SCN neurons to sustain rhythms in the presence of a $\mathrm{VPAC}_{2}$ receptor antagonist indicates that the similar action of GRP in Vipr2 $2^{-1-}$ mice is unlikely to be attributable to developmental abnormalities in GRP-BB ${ }_{2}$ signaling. Given that not all Vipr $2^{-/-} \mathrm{SCN}$ slices sustain MUA rhythms in the presence of GRP and that a $\mathrm{BB}_{2}$ receptor antagonist does not prevent such electrical rhythms in all neurons, it is likely that other intercellular signaling pathways also contribute to the maintenance of rhythmicity in the absence of the $\mathrm{VPAC}_{2}$ receptor. As well as gap junction communication (Jiang et al., 1997; Colwell, 2000; Long et al., 2005), other endogenous SCN neurochemicals such as GABA, prokineticin 2, transforming growth factor $\alpha$, or neuromedin S (Liu and Reppert, 2000; Shirakawa et al., 2000; Cheng et al., 2002; Jobst et al., 2004; Mori et al., 2005) may play important roles in maintaining coherent circadian rhythms in Vipr $2^{-/-}$mice. In addition, because wheel-running activity and sleep states alter SCN cellular activity and behavioral rhythms (Edgar and Dement, 1991; Marchant and Mistlberger, 1996; Yamazaki et al., 1998; Maywood et al., 1999; Schaap and Meijer, 2001; Deboer et al., 2003), and because Vipr $2^{-/-}$mice are wide-ranging in the strength and period of their activity profiles, feedback from behavioral and physiological rhythms could differentially alter SCN rhythmicity in these mice.

This is the first study to reveal fundamental abnormalities in adult SCN physiology at both the individual neuron and neuronal network levels in any mammalian model in which behavioral rhythms are impaired. We demonstrate that SCN slices from Vipr $^{-1-}$ mice expressing coherent behavioral rhythms contain a higher proportion of rhythmic SCN neurons than those prepared from arrhythmic individuals and that, unlike WT animals, expression of these rhythms is partly dependent on $\mathrm{BB}_{2}$ receptor signaling. Remarkably, in the absence of $\mathrm{VPAC}_{2}$ receptor signaling, exogenous GRP promotes SCN cellular rhythms, whereas when this VIP receptor is functional, GRP is detrimental to neural oscillations in these nuclei. Thus, we highlight a potential, unexpected new role for GRP in the regulation of circadian clock function. Identifying the sites and mechanisms of action of GRP in the promotion of rhythmicity merits additional research. These results reinforce recent views that neuronal activity and cell-cell communication are essential for normal pacemaker function and demonstrate how the SCN multioscillator network can adapt to the loss of a key signaling pathway, allowing circadian organization of physiology and behavior.

\section{References}

Aida R, Moriya T, Araki M, Akiyama M, Wada K, Wada E, Shibata S (2002) Gastrin-releasing peptide mediates photic entrainable signals to dorsal subsets of suprachiasmatic nucleus via induction of Period gene in mice. Mol Pharmacol 61:26-34.

Aioun J, Chambille I, Peytevin J, Martinet L (1998) Neurons containing gastrin-releasing peptide and vasoactive intestinal polypeptide are involved in the reception of the photic signal in the suprachiasmatic nucleus of the Syrian hamster: an immunocytochemical ultrastructural study. Cell Tissue Res 291:239-253.

Albers HE, Liou S-Y, Stopa EG, Zoeller RT (1991) Interaction of colocalized neuropeptides: functional significance in the circadian timing system. J Neurosci 11:846-851.

Antle MC, Kriegsfeld LJ, Silver R (2005) Signaling within the master clock of the brain: localized activation of mitogen-activated protein kinase by gastrin-releasing peptide. J Neurosci 25:2447-2454.

Aton SJ, Colwell CS, Harmar AJ, Waschek J, Herzog ED (2005) Vasoactive intestinal polypeptide mediates circadian rhythmicity and synchrony in mammalian clock neurons. Nat Neurosci 8:476-483.

Bouskila Y, Dudek FE (1993) Neuronal synchronization without calciumdependent synaptic transmission in the hypothalamus. Proc Natl Acad Sci USA 90:3207-3210.
Cheng MY, Bullock CM, Li C, Lee AG, Bermak JC, Belluzzi J, Weaver DR, Leslie FM, Zhou QY (2002) Prokineticin 2 transmits the behavioural circadian rhythm of the suprachiasmatic nucleus. Nature 417:405-410.

Colwell CS (2000) Rhythmic coupling among cells in the suprachiasmatic nucleus. J Neurobiol 43:379-388.

Colwell CS, Michel S, Itri J, Rodriguez W, Tam J, Lelievre V, Hu Z, Liu X, Waschek JA (2003) Disrupted circadian rhythms in VIP- and PHIdeficient mice. Am J Physiol Regul Intergr Comp Physiol 285:R939-R949.

Cutler DJ, Haraura M, Reed HE, Shen S, Sheward WJ, Morrison CF, Marston HM, Harmar AJ, Piggins HD (2003) The mouse $\mathrm{VPAC}_{2}$ receptor confers suprachiasmatic nuclei cellular rhythmicity and responsiveness to vasoactive intestinal polypeptide in vitro. Eur J Neurosci 17:197-204.

Deboer T, Vansteensel MJ, Detari L, Meijer JH (2003) Sleep states alter activity of suprachiasmatic nucleus neurons. Nat Neurosci 6:1086-1090.

Edgar DM, Dement WC (1991) Regularly scheduled voluntary exercise synchronizes the mouse circadian clock. Am J Physiol Regul Intergr Comp Physiol 261:R929-R933.

Gribkoff VK, Pieschl RL, Wisialowski TA, van den Pol AN, Yocca FD (1998) Phase shifting of circadian rhythms and depression of neuronal activity in the rat suprachiasmatic nucleus by neuropeptide Y: mediation by different receptor subtypes. J Neurosci 18:3014-3022.

Hannibal J, Fahrenkrug J (2003) Circadian rhythm regulation: a central role for the neuropeptide vasoactive intestinal polypeptide. Am J Physiol Regul Integr Comp Physiol 285:R935-R936.

Harmar AJ, Marston HM, Shen S, Spratt C, West KM, Sheward WJ, Morrison CF, Dorin JR, Piggins HD, Reubi JC, Kelly JS, Maywood ES, Hastings MH (2002) The $\mathrm{VPAC}_{2}$ receptor is essential for circadian function in the mouse suprachiasmatic nuclei. Cell 109:497-508.

Herzog ED, Takahashi JS, Block GD (1998) Clock controls circadian period in isolated suprachiasmatic nucleus neurons. Nat Neurosci 1:708-713.

Herzog ED, Aton SJ, Numano R, Sakaki Y, Tei H (2004) Temporal precision in the mammalian circadian system: a reliable clock from less reliable neurons. J Biol Rhythms 19:35-46.

Hughes AT, Fahey B, Cutler DJ, Coogan AN, Piggins HD (2004) Aberrant gating of photic input to the suprachiasmatic circadian pacemaker of mice lacking the $\mathrm{VPAC}_{2}$ receptor. J Neurosci 24:3522-3526.

Jiang ZG, Yang YQ, Allen CN (1997) Tracer and electrical coupling of rat suprachiasmatic nucleus neurons. Neuroscience 77:1059-1066.

Jobst EE, Robinson DW, Allen CN (2004) Potential pathways for intercellular communication within the calbindin subnucleus of the hamster suprachiasmatic nucleus. Neuroscience 123:87-99.

Kawamoto K, Nagano M, Kanda F, Chihara K, Shigeyoshi Y, Okamura H (2003) Two types of VIP neuronal components in rat suprachiasmatic nucleus. J Neurosci Res 74:852-857.

Lin Y, Stormo GD, Taghert PH (2004) The neuropeptide pigmentdispersing factor coordinates pacemaker interactions in the Drosophila circadian system. J Neurosci 24:7951-7957.

Liu C, Reppert SM (2000) GABA synchronizes clock cells within the suprachiasmatic circadian clock. Neuron 25:123-128.

Long MA, Jutras MJ, Connors BW, Burwell RD (2005) Electrical synapses coordinate activity in the suprachiasmatic nucleus. Nat Neurosci 8:61-66.

Lundkvist GB, Kwak Y, Davis EK, Tei H, Block GD (2005) A calcium flux is required for circadian rhythm generation in mammalian pacemaker neurons. J Neurosci 25:7682-7686.

Maywood ES, Mrosovsky N, Field MD, Hastings MH (1999) Rapid downregulation of mammalian period genes during behavioral resetting of the circadian clock. Proc Natl Acad Sci USA 96:15211-15216.

Marchant EG, Mistlberger RE (1996) Entrainment and phase shifting of circadian rhythms in mice b forced treadmill running. Physiol Behav 60:657-663.

McArthur AJ, Coogan AN, Ajpru S, Sugden D, Biello SM, Piggins HD (2000) Gastrin-releasing peptide phase-shifts suprachiasmatic nuclei neuronal rhythms in vitro. J Neurosci 20:5496-5502.

Moreno D, Gourlet P, De Neef P, Cnudde J, Waelbroeck M, Robberecht P (2000) Development of selective agonists and antagonists for the human vasoactive intestinal polypeptide $\operatorname{VPAC}(2)$ receptor. Peptides 21:1543-1549.

Mori K, Miyazato M, Ida T, Murakami N, Serino R, Ueta Y, Kojima M, Kangawa K (2005) Identification of neuromedin $S$ and its possible role in the mammalian circadian oscillator system. EMBO J 24:325-335. 
Mrugala M, Zlomanczuk P, Jagota A, Schwartz WJ (2000) Rhythmic multiunit neural activity in slices of hamster suprachiasmatic nucleus reflect prior photoperiod. Am J Physiol Regul Intergr Comp Physiol 278:R987-R994.

Nitabach MN, Blau J, Holmes TC (2002) Electrical silencing of Drosophila pacemaker neurons stops the free-running circadian clock. Cell 109:485-495.

Peng Y, Stoleru D, Levine JD, Hall JC, Rosbash M (2003) Drosophila freerunning rhythms require intercellular communication. PLoS Biology $1: 32-40$.

Piggins HD, Cutler DJ (2003) The roles of vasoactive intestinal polypeptide in the mammalian circadian clock. J Endocrinol 177:7-15.

Piggins HD, Rusak B (1993) Electrophysiological effects of pressure-ejected bombesin-like peptides on hamster suprachiasmatic neurons in vitro. J Neuroendocrinol 5:575-581.

Piggins HD, Cutler DJ, Rusak B (1994) Effects of ionophoretically applied bombesin-like peptides on hamster suprachiasmatic nucleus neurons in vitro. Eur J Pharmacol 271:413-419.

Piggins HD, Antle MC, Rusak B (1995) Neuropeptides phase-shift the hamster circadian pacemaker. J Neurosci 15:5612-5622.

Piggins HD, Goguen D, Rusak B (2005) Gastrin-releasing peptide induces c-Fos in the hamster suprachiasmatic nucleus. Neurosci Lett 384:205-210

Quintero JE, Kuhlman SJ, McMahon DG (2003) The biological clock nucleus: a multiphasic oscillator network regulated by light. J Neurosci 23:8070-8076

Reed HE, Meyer-Spasche A, Cutler DJ, Coen CW, Piggins HD (2001) Vasoactive intestinal polypeptide phase-shifts the rat suprachiasmatic circadian clock in vitro. Eur J Neurosci 13:839-845.

Renn SC, Park JH, Rosbash M, Hall JC, Taghert PH (1999) A pdf neuropeptide gene mutation and ablation of PDF neurons each cause severe abnormalities of behavioral circadian rhythms in Drosophila. Cell 99:791-802.

Reynolds T, Pinnock RD (1997) Neuromedin C decreases potassium conductance and increases a non-specific conductance in rat suprachiasmatic neurones in brain slices in vitro. Brain Res 750:67-80.

Romijn HJ, Sluiter AA, Pool CW, Wortel J, Buijs RM (1996) Differences in colocalization between Fos and PHI, GRP, VIP and VP in neurons of the rat suprachiasmatic nucleus after a light stimulus during the phase delay versus the phase advance period of the night. J Comp Neurol 372:1-8.

Romijn HJ, Sluiter AA, Wortel J, Van Uum JF, Buijs RM (1998) Immunocytochemical evidence for a diurnal rhythm of neurons showing colocalization of VIP with GRP in the rat suprachiasmatic nucleus. J Comp Neurol 391:397-405.

Schaap J, Meijer JH (2001) Opposing effects of behavioural activity and light on neurons of the suprachiasmatic nucleus. Eur J Neurosci 13:1955-1962.

Schaap J, Albus H, VanderLeest HT, Eilers PH, Detari L, Meijer JH (2003) Heterogeneity of rhythmic suprachiasmatic nucleus neurons: implications for circadian waveform and photoperiodic encoding. Proc Natl Acad Sci USA 100:15994-15999.

Schmahl C, Bohmer G (1997) Effects of excitatory amino acids and neuropeptide $\mathrm{Y}$ on the discharge activity of suprachiasmatic neurons in rat brain slices. Brain Res 746:151-163.

Shirakawa T, Honma S, Katsuno Y, Oguchi H, Honma K-I (2000) Synchronization of circadian firing rhythms in cultured rat surprachiasmatic neurons. Eur J Neurosci 12:2833-2838.

Sokolove PG, Bushell WN (1978) The chi square periodogram: its utility for analysis of circadian rhythms. J Theor Biol 72:131-160.

Van den Pol AN, Obrietan K (2002) Short circuiting the circadian clock. Nat Neurosci 7:616-618.

Welsh DK, Logothetis DE, Meister M, Reppert SM (1995) Individual neurons from dissociated rat suprachiasmatic nucleus express independently phased circadian firing rhythms. Neuron 14:697-706.

Yamaguchi S, Isejima H, Matsuo T, Okura R, Yagita K, Kobayashi M, Okamura H (2003) Synchronization of cellular clocks in the suprachiasmatic nucleus. Science 302:1408-1412.

Yamazaki S, Kerbeshian MC, Hocker CG, Block GD, Menaker M (1998) Rhythmic properties of the hamster suprachiasmatic nucleus in vivo. J Neurosci 18:10709-10723.

Zoeller RT, Broyles B, Earley J, Anderson ER, Albers HE (1992) Cellular levels of messenger ribonucleic acids encoding vasoactive intestinal peptide and gastrin-releasing peptide in neurons of the suprachiasmatic nucleus exhibit distinct 24-hour rhythms. J Neuroendocrinol 4:119-124. 